\title{
Multi-Scale 1-Bit Compressed Sensing Algorithm and Its Application in Coalmine Gas Monitoring System
}

\author{
Xu Yonggang, Zhang Yi, and Hua Gang
}

\begin{abstract}
It is imperative to reduce load of the underground cable channel in coming Mining of Things for thousands of sensors. Gas data, for example, acts an essential role in mass time-continuous data sets of coalmine monitoring systems, we propose a multi-scale 1-bit compressive sensing algorithm in this paper to effectively compress data according the statistical properties 'regular pattern' of gas data sequence. The algorithm divides input into non-uniform intervals according to the prior attention of the gas monitoring information, then signal decision threshold and the compressed scales depend on the different attention in order to achieve a large scales of compression ratio on redundant data and as much as possible to maintain the sensitive information, comparing with the traditional 1-bit compressive sensing which brings overload quantization distortion during uniform quantization. Satisfactory results obtained by simulation and actual field applications show, which provides a useful reference to similar real monitoring data compression acquisition with compressed sampling.
\end{abstract}

Index Terms-Mining of things (MoT), multi-scale 1-bit compressed sensing (MS 1-bitCS), coalmine safety.

\section{INTRODUCTION}

Internet of Things (IoT) technology has been widely used in open space. 'Perception mine IoT' tries to introduce the IoT technology into mine underground Intranet to capture the real-time multi-dimensional characteristics of a monitored object from the limited space. However, the research is just in its beginning. The large number of data collected by those sensors makes the bandwidth requires rapid increasing in mine IoT, which produces tremendous load on the current communication channel, especially between the channel of source-sensors to fiber looped network as shown in Fig. 1. Part A and part B in gray region, the cable-based channel becomes the bottleneck of restricting the mine IoT while the cost of updating cable is expensive. It's all-important to transform signal sampling into information collection to reduce the massive sampling data with effective data compression method, and release the pressure of the cable-based channel.

Manuscript received May 30, 2013; revised August 1, 2013. This project supported by: 1) National Natural Science Foundation of China No. 51204186. 2) National Science and Technology Support Program No.2012BAH12B02. 3)The Fundamental Research Funds for the Central Universities No. 2010QNB30

$\mathrm{Xu}$ Yonggang and Hua Gang are with School of Information \& Electronic Engineering, China University of Mining and Technology, Xuzhou, Jiangsu, 221008, China (e-mail: feilongxyg@163.com, ghua3323@163.com).

Zhang Yi is with Sience \& Thechnology Center, Huaibei Coalmine Grp. Ltd., Huaibei, Anhui, China (e-mail: zy@ hbcoal.com).

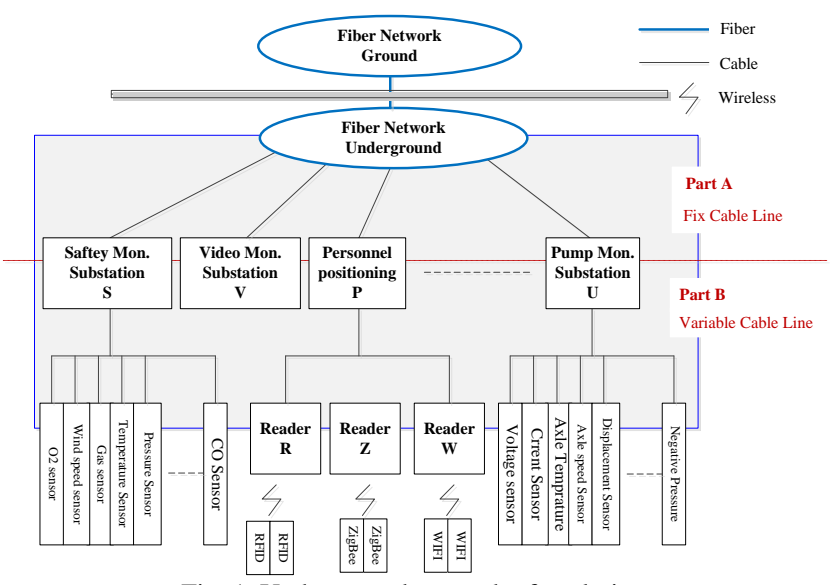

Fig. 1. Underground network of coalmine.

The Compressed/Compressive Sensing/Sampling (CS) theory [1]-[6] recently sprang up in the field of information procession technology is exactly suitable for this special scence, which can effectively overcome the drawbacks of the traditional compression technology based on Nyquist theory. As one important branch of CS theory, 1-bit CS [7]-[11] is paid considerable attention because of its obvious advantage in processing the one-dimensional time series signal, which has become the research focus in this field.

Safety production monitoring system as an essential one of the six systems [12], further more, gas data acts as a core role in saftey monitoring data sets. The gas data is a typical time series data [13], they reflects the real production environment condition, contained with high-dimensional complicated information, which can be implemented in various data analysis, acts as a significant role in several aspects such as system identification, system modeling, decision support and so forth. However, most process data does not evident variation characteristics, but which consumes plenty of network facilities resources during the transmission and storage process. For example, the ' $\mathrm{T} 3$ ' gas sensor named 022A12_II529 of coal face of Yangzhuang coalmine of Huaibei Mining Group Company, when the gas warning threshold and alarming threshold are set to $0.3 \%$ and $1.0 \%$ respectively, more than $95 \%$ of the data collected under $0.3 \%$ is the redundant data, which is not the focus of the monitoring system. In order to ensure the stability and efficiency of the system, this kind of data can therefore be largely compressed.

Gas mutation signal, as a very important monitoring signal, has several characteristics such as mutation, amplitude abnormal, non-sequence and short duration. Due to these characteristics, it is usually implemented in reflecting the potential menace in production environment, which is the indispensable characteristic signal in signal acquisition. Nevertheless, because the signal transient 
variation can not be effectively traced during the 1-bit CS uniform quantization process, the overload quantization distortion is imported, which will lead to the gas characteristic signal distorted or even lost, the signal reconstruction precision is also reduced.To solve the problem 1-bit CS has, we propose the multi-scale nonlinear compression method 'Multi-scale 1-bit CS' based on notability. This method makes the best of prior acknowledge dividing the signal into different scales. By implementing multi-scale compression method, the compression problem in 1-bit CS can be effectively solved.

This paper is structured as following. The Section I states the background. Section II is related to the basic framework and knowledge of compressed sensing and 1-bit compressed sensing. Section III, the detail theory and design of the Multi-scale 1-bitCS based on the scale of concern are introduced, in which the thresholds divide method to optimize designed to closer practical. Then corresponding numerical experiments are presented in Section IV. Result and conclusions are stated in Section $\mathrm{V}$ respectively.

\section{NOTION AND DEFINITION}

\section{A. Traditional Data Compression Method}

The research of traditional data compression algorithms has a history of several decades, according to the statistics study, which have up to 30 to 40 kinds of a variety of data compression methods. On the basis of two main directions, which are distortion of coding and coding modeling, these methods can be separately assorted into several categories. The methods to compress coalmine mass data are classed by [13], [14]: Boxcar method, backward slope method, combination of Boxcar and Backward slope method, SDT method, PLOT method etc. Literature [13] and [14] and the correlate literatures explain the classification and principle of data compression algorithms in details separately, so this article will not repeat them.

\section{B. Compressed Sensing}

CS theory is put forward by D.L Donoho, E. Candès, J. Romberg and T. Tao [1], [4] in 2006. It indicated that, incoherent observation (projection) matrices can be used to project the high-dimension signal to low-dimension space, provided the signal is compressible or is sparse in some region. Afterwards, utilize the small amount of projections to solve an optimization problem, and reconstruct the original signal with high probability. For a compressible signal, it can be reconstructed without distortion by using the sampling rate which is much lower than Nyquist sampling rate. In other words, the sampling rate does not depend on the bandwidth of the signal, but on the structure and contents of information within the signal-transform the signal sampling into information sampling. In fact, some abstract conclusions of CS theory derived from the functional analysis and approximation theory. Sparsity and incoherence are the two main components of CS theory. Former, the premise of CS implementation, is the signal itself. The latter is the condition to decide whether the measurement plan is feasible. The fundamental CS system is shown in Fig. 2. Sparsity representation [1]-[4], [15]-[18], measuring matrix design [16] and reconstruction algorithms [7]-[11], [17], [19] are the core of CS theory.

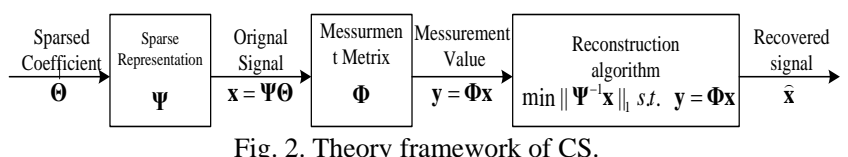

At present, plenty of literatures have given proof of the correlative core theory of what is mentioned above in this article. CS framework theory has aroused great interest in many domestic universities and scientific research institutions. This article aims at the characteristic of coalmine gas monitoring data and introduces the 1-bit CS into practical applications in coalmine.

\section{Multi-Scale 1-BIT CS Algorithm DesigN}

\section{A. Algorithm Description}

In practice, based on years of mine production monitoring, found that for a long time certain types of data have certain 'rules' to follow. Therefore, mine monitoring can obtain abundant a-priori knowledge. Obviously, a-priori knowledge is different according to their operational characteristics in different sectors. But for coalmine safety monitoring dispatch center, the priori knowledge of gas monitoring data can be transformed into a nonlinear, discontinuous multi-scale signal. Moreover, the gas data has the characteristic of non-negative real number field, to meet,

$$
\boldsymbol{x}:=\left\{x_{i}, x_{i} \geq 0, i=1,2, \ldots N\right\}, \boldsymbol{x} \in R^{N}
$$

According to the a-priori knowledge, the gas concentration is divided into different scale ranges, assuming a low concentration of gas [12] in accordance with the scale of concern can be divided as Table I.

TABLE I: ATTENTION DIVISION OF LOW CONCENTRATION OF GAS

\begin{tabular}{cccc}
\hline $\begin{array}{c}\text { Concentrations } \\
\text { of gas }(\%)\end{array}$ & $\begin{array}{c}\text { Security } \\
\text { Level }\end{array}$ & Display Color & $\begin{array}{c}\text { Rules of } \\
\text { attention }\end{array}$ \\
\hline $0 \sim 0.29$ & Safe & Black & $<10 \%$ \\
$0.30 \sim 0.59$ & Warning & Blue & $20 \%$ \\
$0.60 \sim 0.79$ & Warning & Orange & $40 \%$ \\
$0.8 \sim 1.00$ & Warning & Purple & $80 \%$ \\
$>1.00$ & Alarm & Red,flashing,voice & $100 \%$ \\
\hline \hline
\end{tabular}

Obviously, the gas concentration is divided into a plurality of intervals ranging scale interval, the attention given to different scales, with a mathematical description is,

$$
\begin{aligned}
& A(x)=A_{i} \\
& s_{i-1} \leq x<s_{i}
\end{aligned}
$$

where $A_{i}, i \subset\{1,2, \ldots, N\}$ are different segments of attention, $S_{i}$ is the boundary of threshold segmentation, and,

$$
\left\{\begin{array}{l}
s_{i}-s_{i-1}=l_{i}>0 \\
l_{i-1} \neq l_{i}
\end{array}\right.
$$


where $l_{i}, i \subset\{1,2, \ldots, N\}$ denotes the scale for different interval, according to the amount of information, let $p(x)$ the probability of $x$, we set the amount of information is proportional to the scales of attention with the relationship,

$$
A(x)=-\ln p(x)
$$

Since the distribution of eigenvalues are randomly uncertain, assume that:

$$
f(x)=p^{\prime}(x)=\frac{1}{a-b}
$$

where $f(x)$ is the probability density, supposing it uniformly distributed random probability event, $x_{i} \in[a, b]$ indicates the signal dynamic range, and then assume quantized output satisfies,

$$
q(A):=\frac{\eta}{A}
$$

where $\eta$ is a curve correction constant, then the quantized output satisfies,

$$
q(A(x))=\frac{\eta}{A(x)}
$$

The compression ratio denoted by $\delta(x)$ can be obtained,

$$
\delta(x):=q^{\prime}(x)=-\frac{\eta A^{\prime}(x)}{A^{2}(x)}
$$

Let (2), (4), (5) into (8),

$$
\begin{aligned}
\delta_{i}= & q^{\prime}(x)=-\frac{\eta A^{\prime}(x)}{s_{i-1} \leq x<s_{i}} \\
& =-\frac{\eta}{A_{i}^{2}}[-\ln p(x)]^{\prime} \\
& =\frac{\eta \cdot p^{\prime}(x)}{A_{i}^{2} \cdot p(x)} \\
& =\frac{\eta}{(a-b)} \cdot \frac{1}{A_{i}^{2}} \cdot \frac{1}{p(x)}
\end{aligned}
$$

Because $p(x)$ is a constant with a-priori probability, and $p(x)=c, c \in R^{+}$, assume const $\lambda(\lambda>0)$,

$$
\lambda=\frac{\eta}{c(a-b)}
$$

then (9) simplifies to,

$$
\delta_{i}=g^{\prime}(x)=\frac{\lambda}{A_{i}^{2}} \quad x \in\left[s_{i-1}, s_{i}\right)
$$

Thus, the quantization step of signal is inversely proportional to the square of the threshold interval signal attention, obviously this is consistent with the practical enterprise data governance, the higher the attention of data, the smaller the quantization step, the smaller the compression ratio, even a compression ratio of 1 (no compression); for small attention data, man can give a high compression. But in practice, data with small attention has an absolute high probability; sensitive data has only small proportion, which provides possibility for high compression ratio. In order to facilitate the calculations in practice, we use a piecewise linear approximation method to segmented set the threshold, the quantization step obviously is no more a constant.

\section{B. MS 1-bitCS Algorithm Flow}

According the analysis, we improved the 1-bitCS algorithm in the multi scales based literature [8] as following.

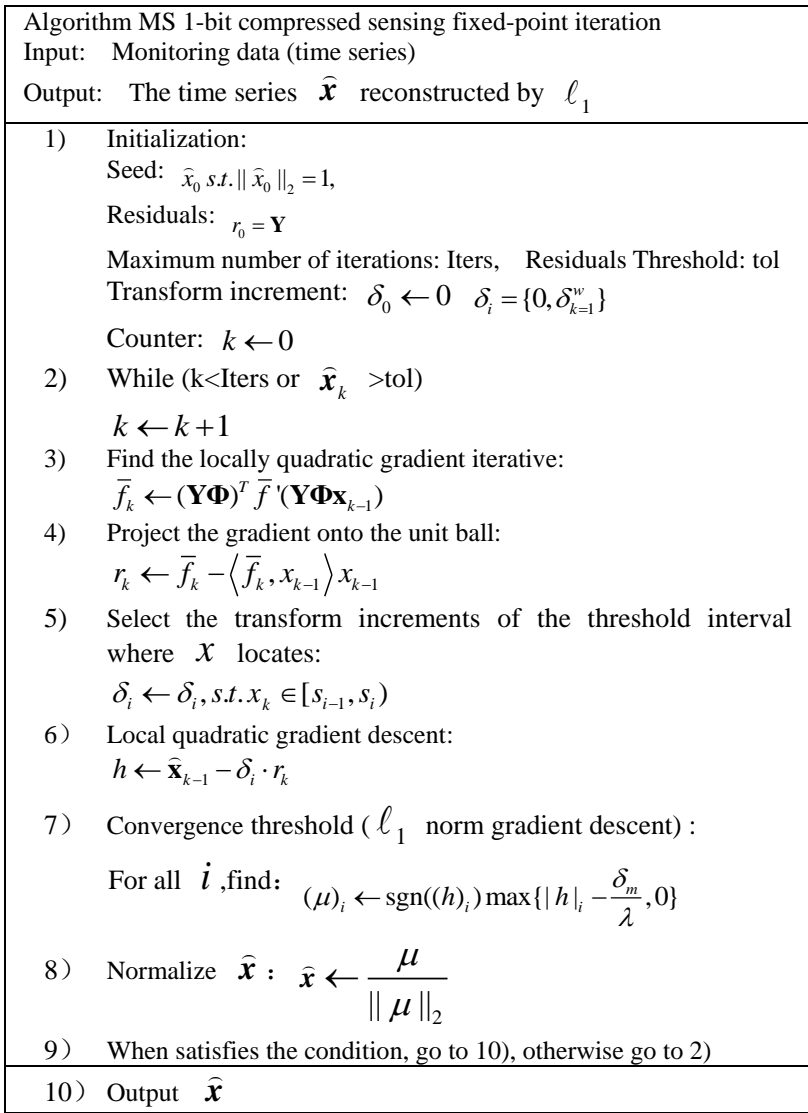

\section{EXPERIMENTS AND APPLICATIONS}

\section{A. Data Sources}

This paper selects part of the data of Huaibei Mining Group Young Mine gas monitoring points HB05_022A12 2013.4.2 2013.4.12 as the original data shown as Fig. 7, which reflects the true changes of underground gas concentration shown in Fig. 3 red line. It shows both of the slowly varying gas data, the gas abnormal data, and the equipment condition monitoring data (gas sensor calibration) [12].

\section{B. Experimental Environment}

This experiment uses Matlab2010b, 2G RAM, Intel dual core $1.7 \mathrm{GHzCPU}$, WINXP operating system as a simulation platform, we use regularized identity dictionary based on multi-scale attention, and identity matrix as measurement matrix, respectively with hard threshold gradient iteration, 
and orthogonal matching pursuit (OMP) and compressed sensing orthogonal matching pursuit (CoSaMP) algorithm combining a-priori knowledge to reconstruct the signal, the simulation shown in Fig. 3 to Fig. 6.

\section{Analysis of Experimental Results}

To compare the performance between 1-bit CS literature [8] and our proposed method when overload quantization, experience selects $\Delta=\delta_{m} / \lambda=0.05$, and about 23 miniutes samples form original data set, it is shown in Fig. 7 where like a maxmum amplitude 'pulse',which exactly includes 1401 samples indeed. We compare 1-bit CS and MS 1-bit CS separately in three aspects which are sparse representation of the signal, reconstruction error and reconstruction results, shown in Fig. 3 to Fig. 6. The original signal,1-bit CS and proposed method sparse projections is

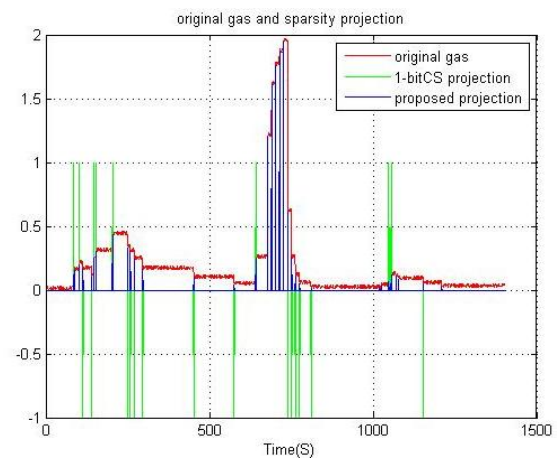

Fig. 3. Signal sparse representation under two kinds of algorithms.
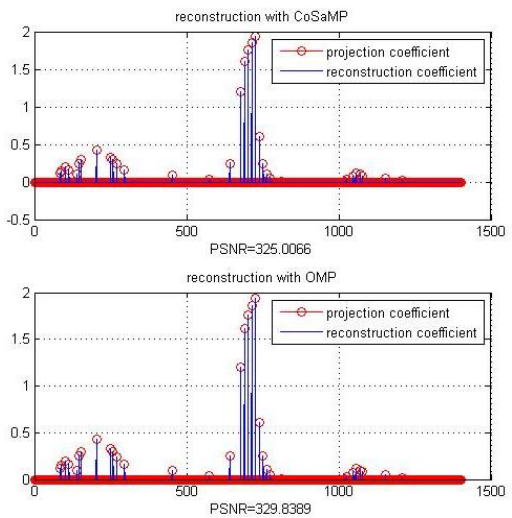

Fig. 5. OMP and CoSaMP reconstruction coefficient. shown in Fig. 3 respectively, directly non-uniform equivalent projects for large coefficients according to priori attention, which is different from the traditional 1-bit CS incremental projection. Seen from the projection coefficient, the protection coefficients of MS 1-bitCS are more than which of traditional methods in the place where information changes significantly, while exhibits the same performance for the sparse small signal.

The error curve shown in Fig. 4 and the reconstruction curve shown in Fig. 6 reflect visually, when signal mutation occurs, due to the restriction of quantization $\Delta=\delta_{\mathrm{m}} / \lambda$ (constant), 1-bit CS has a great error with the original signal, which results in abnormal loss of information; while MS 1-bitCS can remain this error in a very small range (0.02), $\mathrm{PSNR}=31.8 \mathrm{~dB}, \mathrm{MSE}=0.106$;

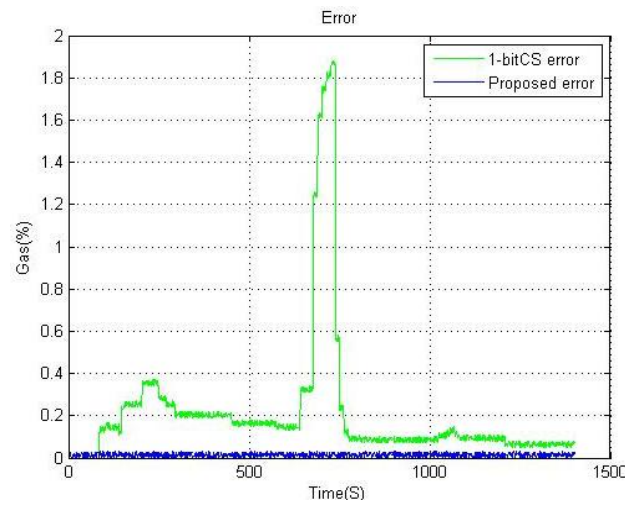

Fig. 4. Signal sparse representation error.

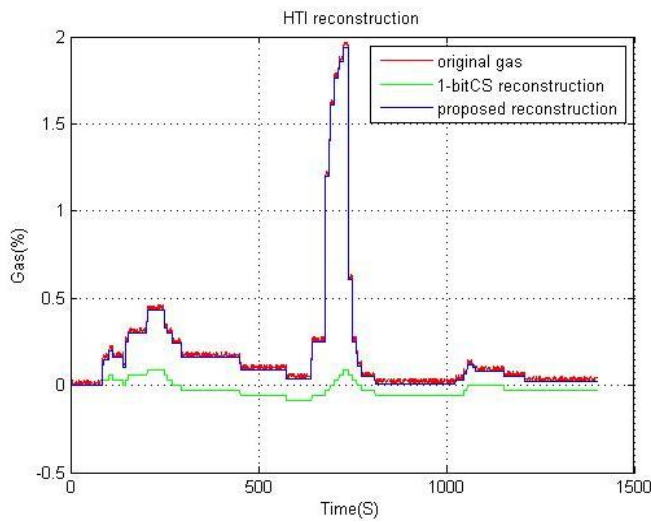

Fig. 6. Signal reconstruction with 1-bit CS and proposed.

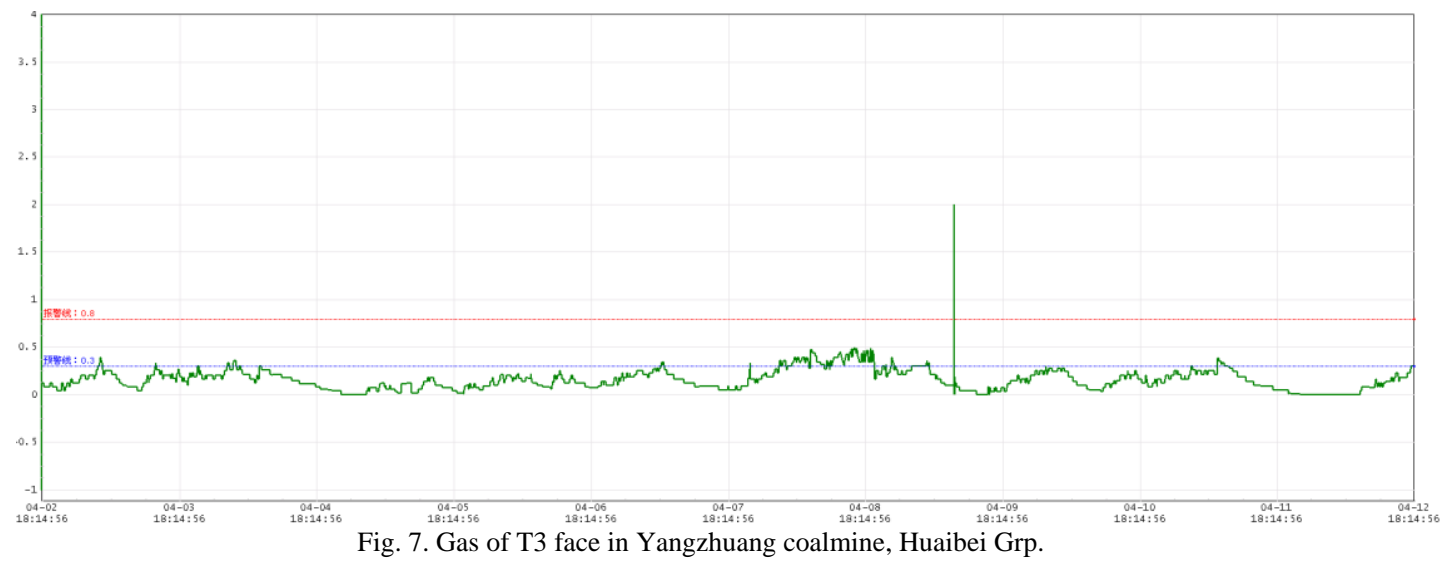

When $\Delta$ is reduced, the error 1-bit CS quantified on 'exception' data increases further, and the error of MS 1-bitCS is reduced.

Using OMP and CoSaMP shown in Fig. 5, select
$\frac{k_{m}}{N}=0.2$ as the default sparse threshold $\left(k_{m}\right.$ is the default maximum possible sparse value, $N$ is the length of input 
signal, use $N=1401$ in this experiment), get the real sparse value $k=33$. When reconstruct the sparse projection coefficients using the random Gaussian observation $M=O(k(3+\log (N / k))=165$, PSNR can achieve $329 \mathrm{~dB}$ and $315 \mathrm{~dB}$ respectively, to realize exact reconstruction of the coefficients. Considering the quantization noise introduced error, the PSNR of these two methods of reconstruction curve can achieve $44.6 \mathrm{~dB}$ and $43.3 \mathrm{~dB}$ and exactly reconstruct the original curve, but the time of reconstruction should be longer than that of the hard threshold gradient projection method. As is shown in Fig. 7, this algorithm shows good performance in the actual use in Huaibei Mining Group's production.

\section{CONCLUSION}

According to the prior acknowledge, the monitoring data of different intervals can be divided into different scaled $\delta$, then measured the segmented error separately, and implemented the delaminating compression in data with different attention, in order to radically realize high compressive ratio in safety data and no compression in sensitive warning data.

Through the simulation calculation, the reconstructed PSNR of high attention projection coefficient using the algorithm was up to $334 \mathrm{~dB}$, then the accurate reconstruction was realized. Regarding the imported error of quantization noise, the construction curve was up to $44.6 \mathrm{~dB}$, that suffice to reconstruct the original curve and meet the need of the enterprise.

It is worthwhile to explain that, by using OMP and CoSaMP methods to reconstruct the coefficient, when the sparsity $k<<\log \frac{k}{N}<0.03, \quad M=O(k \log (N / k)) \quad$ cannot effectively reconstruct spasity, the reconstruction probability is high-low. Within the 50 experiments, only 1 successful reconstruction, the probability is $2 \%$. When amend the formula to $M=O(3 k+\log (N / k))$, within 30 experiments, 25 successful reconstructions, the probability is $83.3 \%$. Therefore the observation matrix design with extreme sparse condition still needs to be analyzed theoretically in the future.

\section{ACKNOWLEDGMENT}

This work specially thanks for communications office, dispatch center etc., Huaibei Coalmine Group, Anhui Prov.

\section{REFERENCES}

[1] E. Candès, "Compressive sampling," International Congress of Mathematics, Madrid, Spain, vol. 3, pp. 1433-1452, 2006.

[2] E. Candès, J. Romberg, and T. Tao, "Robust uncertainty principles: Exact signal reconstruction from highly incomplete frequency information," IEEE Trans. On Information Theory, vol. 52, no. 2, pp 489-509, 2006.

[3] E. Candès and J. Romberg, "Quantitative robust uncertainty principles and optimally sparse decompositions," Foundations of Comput. Math., vol. 6, no. 2, pp. 227-254, 2006.

[4] D. Donoho, "Compressed sensing," IEEE Trans. On Information Theory, vol. 52, no. 4, no. 1289-1306, 2006.

[5] G. M. Shi et al., "Advances in theory and application of compressed sensing," ACTA ELECTRONICA SINACA, vol. 37, no. 5, pp. 1070-1081, 2009.
[6] L. C. Jiao, S. Y. Yang, F, Liu, and B. Hou, "Development and prospect of compressive sensing," ACTA ELECTRONICA SINICA, vo1. 7, pp. 1651-1662, 2011.

[7] Y. Plan and R. Vershynin, "Robust 1-bit compressed sensing and sparse logistic regression: a convex programming approach," IEEE Trans. on Information Theory, vol. 59, no. 1, pp. 482-94, 2013.

[8] T. Petros et al., "1-Bit compressive sensing," in Proc. The 42nd Annual Conf. on Information Sciences and Systems, 2008, CISS2008, pp. 16-21.

[9] T. Y. Zhou and D. C. Tao, "1-bit Hamming compressed sensing," in Proc. IEEE International Symposium on Information Theory Proceedings, ISIT 2012, 2012, pp. 1862-1866.

[10] A. Clemente, et al., "1-Bit reconfigurable unit cell based on PIN diodes for transmit-array applications in X -band," IEEE Trans. on Antennas and Propagation, vol. 60, no. 5, pp. 2260-2269, 2012.

[11] M. Yan, Y. Yang, and S. Osher, "Robust 1-bit compressive sensing using adaptive outlier pursuit," IEEE Trans. on Signal Processing, vol. 60, no. 7, pp. 3868-3875, 2012

[12] National Security Supervision Bureau., Coal Mine Safety Regulations 2012 - new revision, Coal Industry Press, March 2011.

[13] G. Hua, "Key technology research on synthetically dispatching system of safety and production in coal mine," CUMT Library, 2002.

[14] K. Sayood, Introduction to Data Compression, 3rd ed. Posts and Telecom Press, 2009. 2.

[15] B. Kashin, "The widths of certain finite dimensional sets and classes of smooth function," IZV AKad Nauk SSSR, vol. 41, no. 2, pp. 334-351, 1977.

[16] E. Candès and T. Tao, "Near optimal signal recovery from random projections: Universal encoding strategies?" IEEE Trans. Info. Theory, vol. 52, no. 12, pp. 5406-5425, 2006.

[17] D. L. Donoho, Y. Tsaig, I. Drori, and J. L. Starck, "Sparse solution of underdetermined systems of linear equations by stagewise orthogonal matching pursuit," IEEE Trans. on Information Theory, vol. 2, no. 58, pp. 1094-121, 2012

[18] M. Elad and A. M. Bruckstein, "A generalized uncertainty principle and sparse representation in pairs of bases," IEEE Trans. Inform Theory, vol. 48, no. 9, pp. 2558-2567, 2002.

[19] E. Candès and J. Romberg, "Practical signal recovery from random projections," in Proc. Storage and Retrieval for Image and Video Databases, 2004.

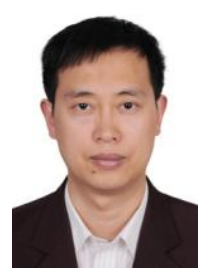

Xu Yonggang was born in February 1977 in Pingchang, Sichuan, China. He received the B.S. and M.S degree in Electronic information from School of Information \& Electronic Engineering, China University of Mine \& Technology, Xuzhou, Jiangsu, China in 2001 and 2006 respectively. He is a Ph.D. Candidate and mainly study in information fusion, Mine Internet of Things, coalmine communication

He joined the Department of Information \& Electronic Engineering, China University of Mine \& Technology, Xuzhou, Jiangsu in 2001. His research interests include coalmine signal processing, as well as its applications in enterprises, Mine Internet of Things, compressed sensing, and variational image processing.

Dr. Xu won the NSFC CAREER award in 2012.

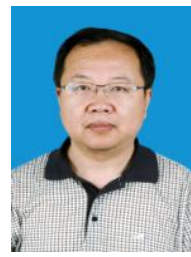

Hua Gang is a professor. He was born in May 1963 in Wuxi, Jiangsu, China. He received the B.S. in Industrial Automation, Southeast University, Nanjing, M.S and Ph.D. degree in Electronic information from School of Information \& Electronic Engineering, China University of Mine \& Technology, Xuzhou, Jiangsu, China in 2000 and 2004 respectively. He mainly studys in DSP, machine vision, information fusion, image processing.

He joined the Department of Information \& Electronic Engineering, China University of Mine \& Technology, Xuzhou, Jiangsu in 1984. His research interests include coalmine signal processing and its applications in enterprises, DSP, machine vision, information fusion, and variational image processing, intelligent computing as well.

Prof. Hua is member of automation institution, China Electronic Chamber of Commerce networking technology product applications professional advisory committee.About 10 honours won and more than 90 papers published, and indexed by EI over 10 papers. 